\title{
Improvement of QoS Contained by AODV Routing Protocol On the Basis of Varying Queue Length and Dynamic TTL Value in MANET
}

\author{
Shweta yadav, Vivek Richhariya \\ MTech student CSE Deptt.,Assistant Professor CSE Deptt. LNCT Bhopal, LNCT Bhopal
}

\begin{abstract}
A Mobile ad-hoc network (MANET) is a network, self-configuring, proficient of self-directed functioning, quickly deployable and operates without infrastructure. MANET operates without any centralized administration. The nodes are self configuring, independent, quickly deployable. Nodes are movable since topology is dynamic and they have restricted computing resources. We know that routing protocols make an important role for improving Quality of Service $(Q \circ S)$ in Mobile Ad hoc Network. The reactive AODV routing faces problems like long route, time delay, mobility and many other while routing. In this work the quality of $A O D V$ routing protocol has been improved to enhance the routing capability. In this paper the performance of normal AODV routing protocol are improves on the basis of QoS. Here we consider the particular TTL Value and dynamic threshold value for established the connection in long route and also measure the varying queue length technique by that if the node buffer size is full then no packet is drop form queue, it means the size of queue is varying according to data. The dynamic TTL value established the connection with long route receiver and the varying queue minimizes the packet loss. The quality of Routing protocols should integrate QoS metrics in route finding and maintenance, to support end-to-end QoS. The performance of improves AODV protocol is measures on the basis of performance metrics.
\end{abstract}

Keywords: MANET, QOS, TTL, AODV, Queue length.

\section{INTRODUCTION}

Mobile Ad Hoc Networks (MANETs) is a class of wireless networks that have been researched widely over the recent years [1]. MANETs do not require the support of wired access points or base stations for intercommunication. A mobile ad hoc network, unlike a static network, has no infrastructure. It is a collection of mobile nodes where communication is established in the absence of any fixed foundation. The only possible direct communication is between neighboring nodes. Therefore, communication between remote nodes is based on multiple-hop. These nodes are dynamically and arbitrarily located in such a manner that the interconnections between nodes are capable of changing on a continual basis. MANETs are self-configuring; there is no central management system with configuration responsibilities. All the mobile nodes can communicate each other directly, if they are in other's wireless links radio range. In order to enable data transfer they either communicate through single hop or through multiple hops with the help of intermediate nodes. Since MANETs allow ubiquitous service access, anywhere, anytime without any fixed infrastructure they can be widely used in military battlefields, crisis management services, classrooms and conference halls etc. MANETs ad-hoc fashion networking developments lead to development of enormous multimedia applications such as video-on-demand, video conferencing etc. Routing in mobile ad hoc networks and some fixed wireless networks use multiple-hop routing. Routing protocols for this kind of wireless network should be able to maintain paths to other nodes and, in most cases, must be handle changes in paths due to mobility.

The Transmission control protocol (TCP) is one of the most widely used end-to-end transport layer protocol in the internet today.TCP ensure reliable data transfer over unreliable networks. The TCP performs three major tasks i) Connecton Establishment ii)Data Transfer iii) Connection Termination. Major problems of TCP degradation in mobile networks are mobility, high bit error rate, Scalability, hidden and exposed node problem etc. At present traditional wired network are being replaced by wireless networks. The main reasons may be the tremendous technical growth in the wireless communication area and the reducing cost of wireless devices.

However, there still remains a significant challenge to provide QoS solutions and maintain end-to-end QoS with user mobility. Most of the conventional routing protocols are designed either to minimize the data traffic in the network or to minimize the average hops for delivering a packet. [1]. Even some protocols such as Ad-hoc On demand Distance Vector (AODV) [2], Dynamic Source Routing (DSR) [3] and On-demand Multicast Routing Protocol (ODMRP) [4] are designed without explicitly considering QoS. When QoS is considered, some protocols may be unsatisfactory or impractical due to the lack of resources and the excessive computation overhead. QoS routing usually involves two tasks: collecting and maintaining up-to-date state information about the network and finding feasible paths for a connection based on its QoS requirements. [5] To support QoS, a 
service can be characterized by a set of measurable pre specified service requirements such as minimum bandwidth, maximum delay, maximum delay variance and maximum packet loss rate.

\section{About AODV Routing Protocol}

Ad hoc on Demand Distance Vector (AODV) [2] is an on-demand routing protocol that builds routes only when desired. It makes use of sequence numbers to ensure the freshness of routes. To find a path to a destination, a node using AODV broadcasts a route request (RREQ) packet on the bases of Fixed Time to Live (TTL) value. The RREQ contains the node's IP address, current sequence number, broadcast ID and most recent sequence number for the destination known to the source node. The destination node on receipt of RREQ, unicasts a route reply (RREP) packet along the reverse path established at the intermediate nodes during the route discovery process. In case of a link failure or expires TTL value a route error (RERR) packet is sent to the source and destination nodes. By the use of sequence numbers, the source nodes are always able to find new valid routes.

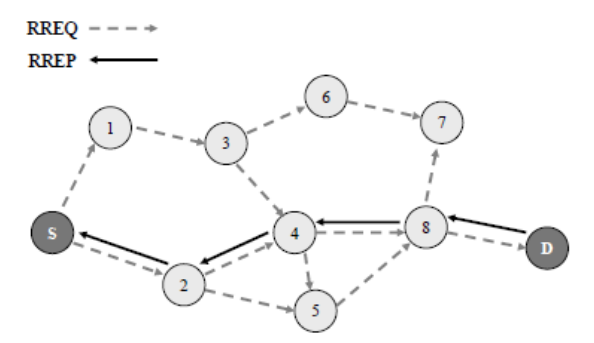

Fig. 1 AODV Route Discovery Procedure

\section{LITERATURE SURVEY}

In [6], the authors proposed an extension to AODV to support QoS, assuming the availability of some stationary links in the network. The authors introduced the notion of node stability, based on a node's history, which incorporated both a node's mobility and its packet processing ratio. Only stable nodes were considered for routing. However, the authors did not consider the impact that unpredictable link failures would have on rerouting.

In [7] authors have proposed a stable, weight-based, on-demand routing protocol. The "weight" carried in the protocol messages used to select stable routes is based on three components: Route Expiration Time (RET), which is the predicted time of link breakage between two nodes due to mobility, Error Count (EC), which captures the number of link failures due to mobility, and Hop Count (HC). The authors have assumed that all nodes are synchronized via a Global Positioning System (GPS), so that two adjacent nodes may predict the RET. While the proposed scheme may combat against link breaks due to mobility, link breaks due to the draining node energy is a factor that also must be accounted for when computing weights for stable routing.

In [8], the authors have proposed a stable route selection scheme based on Link Expiration Time Threshold (LETth). The Link Expiration Time (LET) is computed based on a prediction of neighbor mobility. LET computation needs to know the position of the neighbors, and hence requires periodic topology updates. However, the authors have not considered the impact that unpredictable link failures would have on re-routing.

In [9], the authors proposed a new metric, Energy- Drain-Rate, which is defined as the rate at which energy is consumed at a given node at time t. The corresponding cost function is defined as:

$$
\mathrm{C}_{\mathrm{R}}=\min T_{r}^{i}(t) \text {, where } T_{r}^{i}(t)=\frac{E_{r}^{i}(t)}{D_{r}^{i}(t)}
$$

Where $D_{r}^{i}(t)$ and $E_{r}^{i}(t)$ are the drain rate and the residual battery power respectively, of node i at time $t$ along the path $\mathrm{r}$. Thus the life-time of a path $\mathrm{R}$ is determined by the minimum $T_{r}^{i}(t)$ along that path. The Minimum Drain Rate (MDR) mechanism selects the route with maximum life-time. Each node monitor its energy consumption during a given past interval and maintains the drain rate value using an exponential weighted moving average. The proposed MDR algorithm attempts to select the best possible stable route for a given source and destination. The periodic route update used in MDR, however, soon becomes costly, as it increases control overhead and degrades performance at higher network loads. From the proposals reviewed so far [8] it is clear that there is a need for a routing protocol that can provide stability to the routes selected for routing QoS-enabled applications, and also has mechanisms for fast re-routing to tackle unpredictable link breakages. Furthermore, for the scheme to be scalable, the stability should come at minimum or no overhead. In what follows, we propose modifications to the AODV protocol that, with high probability, provide routes that are stable for a session duration, and that also incorporate a fast make-before- break mechanism.

In [10] QoS routing has received attention recently for providing QoS in wireless ad hoc networks and some work has been carried out to address this critical issue. Here, we provide a brief review of existing work addressing the QoS routing issues in wireless ad hoc networks. In general, QoS routing can be classified into two 
basic paradigms: source QoS routing and hop-by-hop QoS routing. Hereafter, the term routing will refer to QoS routing unless otherwise specified. With source routing, the source node of a communication request locally computes the entire constrained path to the intended destination with the global state information that it locally maintains. Gathering and maintaining global state information can introduce excessive protocol overhead in dynamic networks and thus have the scalability issue. Moreover, the calculation of constraint(s)-based routes would be computationally intensive for the calculating nodes. The predictive location- based QoS routing protocol. This protocol is mainly to alleviate the scalability issue with respect to communication overhead in implementing source routing. Instead of disseminating the state of each link network wide, each node broadcasts its node status (including its current position, velocity, moving direction, and available resources on each of its outgoing links) across the network periodically or upon a significant change. With such information, at any instant each node can locally depict an instant view of the entire network. To accommodate a QoS request, the source locally computes a QoS satisfied route (if available) and route data packets along the calculated path. Moreover, the source can predict route break and predicatively compute a new route before the old route breaks by using the global state it stores. This routing protocol is suitable for providing soft QoS in small or mediumsized networks wherein mobile hosts are equipped with Global Positioning System (GPS) receivers and their moving behavior is predictable.

The routing protocols for MANETs may be broadly classified as table driven protocols $[11,12]$ and ondemand driven protocols $[13,14]$. Table driven protocols need to maintain the global routing information about the net-work in every mobile node for all the possible source-destination connection and acquire to exchange routing information periodically. This kind of protocol has the property of lower latency and higher overhead.

\section{Problem Statement}

MANET is form of distributed network with infrastructure less environment that endorse unreliable and low quality of data service so our aim to provide more reliable as compare to exiting routing protocol services using enhancement of routing protocol and transport layer scheme and improve quality of service of communication.

\section{PROPOSED SCHEME:}

Here we proposed AODV updated scheme for routing over head minimization as well as queue variation scheme for data drop minimization and retrieve enhancement result of updated routing scheme, in this algorithm initially we set time to live as 7 millisecond and threshold time to live as dynamic and one constant value and through that value we broadcast route request packet and find out genuine receiver and dynamic threshold scheme useful for longer route request response time case, after that scheme we apply queue variation base technique for data drop minimization in that scheme we check queue limit if limit value is full so we cannot drop data rather than increases queue limit by one and save data into queue. That algorithm gives better quality of service as well as maximum performance as compare to existing AODV routing system.

Set TTL_START 7 //time to live $7 \mathrm{~ms}$

set TTL_THRESHOLD ; //Threshold Dynamic Setting

define TTL_INCREMENT 2 // increment by $2 \mathrm{~ms}$

define TTL la 5 //Constant Value

flood-route(AODV,S,R) //(protocol, source, receiver)

$\{/$ here ttl limit not constant so vary according to dynamically

If (node in range $\& \&$ next-hop!=R)

$\{$ search next-hop; If (next-hop!=R \&\& loop free)

\{

Continue tile all radio range nodes route packet receives

\}

\}

Else $\{$ Receiver found;

Data_send();

\}

Data_send(S,R,data) // sending case drop minimization

\{

Generate application data;

Sender send's data through computed path;

intermediate node

Check (Q-limit of I node's) // dynamic variation Q scheme at

$$
\text { If (Q-limit }==\text { Full) }
$$




\author{
$\{$ increment-Q; \\ \} \\ Store incoming data; \\ Receiver receives data from I node; \\ Send ACK to sender S; \\ \}
}

VI. Simulation ENVIRONMENT

The network simulator (ns-2) [15] is a discrete network simulator targeted at network researching. Ns-2 originated in 1989 as a variant of the REAL network simulator. As a part of the Virtual Inter Network Testbed (VINT) project at the University of California in Berkley. The project was supported by Defense Advanced Research Projects Agency (DARPA) in 1995. Ns-2 is an object-oriented simulator with substantial support available for simulation of TCP, routing, and multicast protocols, initially intended for wired networks, but the Monarch Group at Carnegie Mellon University (CMU) have extended ns-2 to support wireless networks.

\title{
VII. Simulation ResUlts
}

This section represents the results that has generated after applying the proposed scheme and compare the performance with normal AODV protocol.

\section{A. PDR Analysis}

Packet delivery ratio is the calculation of total percentage of packets that are successfully delivered and receive in network. This graph represents Packet Delivery Ratio (PDR) Analysis in case of normal AODV protocol and proposed enhanced QoS based routing protocol. Here the performance of proposed QoS based AODV protocol is better as compare to previous AODV. Now in time about after 30 second the performance of proposed enhance AODV protocol is maintaining the improvement up to end of simulation. The packet percentage in case of normal AODV routing is about $91 \%$ but in case of QoS based routing is about $95 \%$, it means the concept of dynamic changing TTL value is definitely improving the routing efficiency and also enhanced the performance of routing protocol.

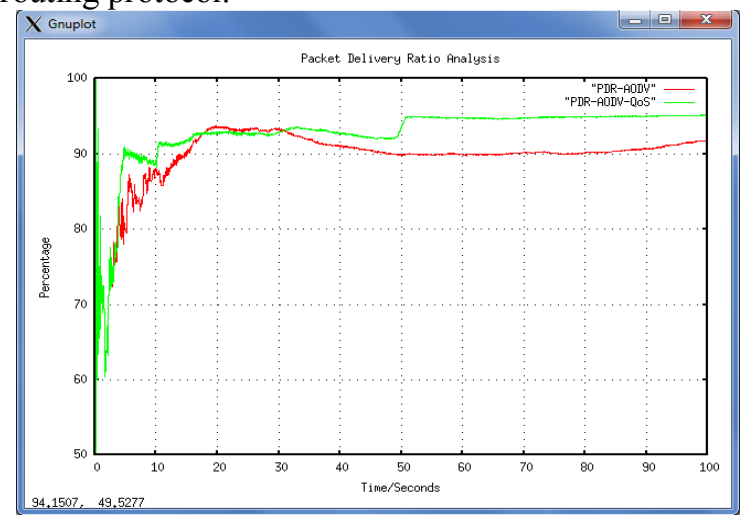

Fig. 2 PDR Analysis

\section{B. Routing Load Analysis}

Routing overhead is one of the important factor to measure the performance of routing protocol in ad hoc network. The routing packets or connection establishment packets are required to maintain the connection in between sender and receiver after that the data delivery is starting. Due to the dynamic nature the routing overhead minimization is the challenging task in ad hoc network. In this graph the routing overhead in case of proposed enhanced QoS based AODV protocol is very efficient as compare to normal AODV routing protocol. The lower value of routing overhead is shows the better performance. In proposed approach only about 5000 routing packets are deliver in network but in case of normal AODV routing the above 9000 routing packets are deliver in network. It means that the performance of proposed protocol is much better than normal AODV and the more number of data packets are deliver in network in case of enhanced QoS AODV that is by prove mentioned in overall summery table 2. 


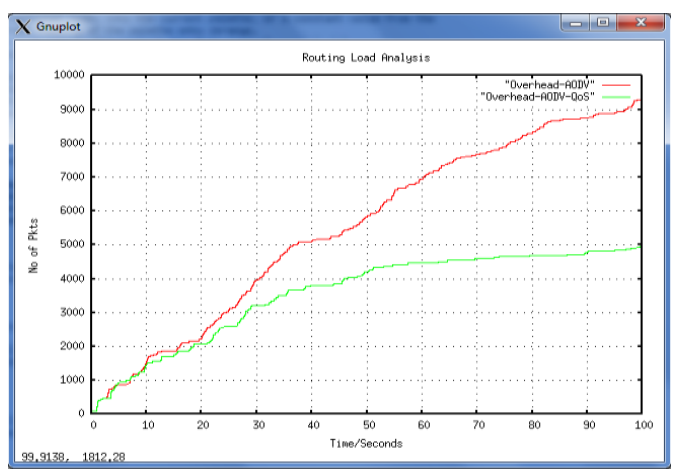

Fig. 3 Routing Load Analysis

C. Throughput Analysis

Throughput is represents the number of packets sends or receives in network in per unit of time. It means the through put represents the successful delivery or receiving with respect to time. In this graph the throughput performance of proposed enhance QoS based AODV routing protocol and normal ADV routing protocol is measured, here we observe that initially at time from starting to 15 seconds the performance of proposed protocol are better but after that the AOSV is better up to 50 seconds and then again after 50 seconds the performance of proposed protocol are better. It means the concept of dynamic TTL value is definitely improves the performance of routing protocol. Because of the higher routing overhead shown in figure 3 degrades the network performance of normal AODV routing protocol. If the more number of routing packets are deliver in network means data packets are waiting for connection establishment then packets sending capability affected and performance of network are degrade.

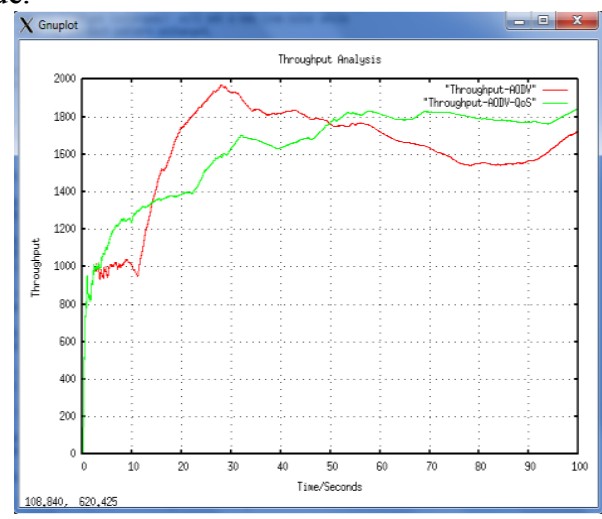

Fig. 4 Throughput Analysis

\section{UDP packet Analysis}

Use Datagram Protocol (UDP) performance is measure here due to their unreliable behaviour it means because of their connection less property by that no connection establishment are happed here, directly data delivery is started in network. Due to connection less behaviour the possibility of packet loss is more, it means the performance of this protocol is depend on network conditions. In this graph the performance of proposed and normal AODV routing protocol is nearly equal from starting to 50 second. But after that in case of proposed enhanced QoS based UDP packet receiving capability is increases continuously up to end of simulation as compare to normal AODV. In proposed case about more than 300 packets are receive in network but in case of normal AODV only about 220 packets are receive in network. It means the proposed protocol is more reliable than previous.

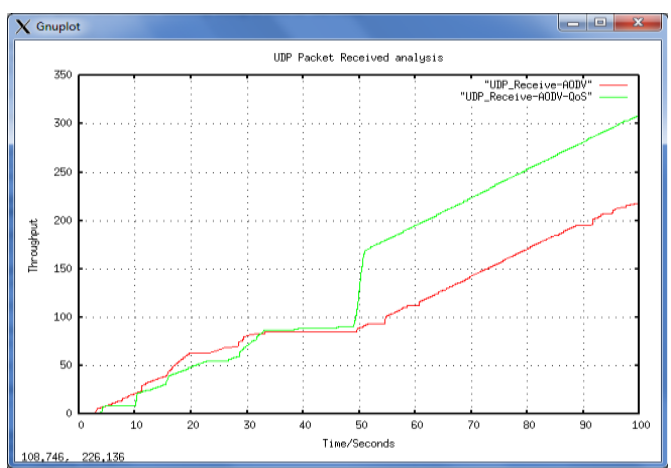

Fig. 5 UDP packet analysis 


\section{E. Drop Analysis due to Different reasons}

There are many reasons of packet dropping in MANET like collision, congestion, link breakage etc. This table 1 represents the different drop reasons. This table 1 show how many packets are drop in network in case of proposed enhanced QoS based AODV protocol and normal AODV routing protocol and also show the drop percentage with every drop reason.

Table 1 Drop Analysis under AODV and AOD-QoS Time

\begin{tabular}{|c|c|c|c|c|}
\hline DROP Analysis & \multicolumn{2}{|c|}{ AODV } & \multicolumn{2}{|c|}{ AODV-QOS } \\
\hline Drop from ARP & 56 & $0.28 \%$ & 18 & $0.11 \%$ \\
\hline Drop from IFQ & 79 & $0.39 \%$ & 35 & $0.22 \%$ \\
\hline Drop from $\mathrm{CBK}$ & 256 & $1.27 \%$ & 111 & $0.71 \%$ \\
\hline Drop from TOT & 0 & $0.00 \%$ & 0 & $0.00 \%$ \\
\hline Drop from NRT & 150 & $0.74 \%$ & 19 & $0.12 \%$ \\
\hline Drop from END & 23 & $0.11 \%$ & 17 & $0.11 \%$ \\
\hline Drop from DUP & 0 & $0.00 \%$ & 0 & $0.00 \%$ \\
\hline Drop from RET & 470 & $2.33 \%$ & 240 & $1.53 \%$ \\
\hline Drop from BSY & 6 & $0.03 \%$ & 13 & $0.08 \%$ \\
\hline Drop from SAL & 0 & $0.00 \%$ & 0 & $0.00 \%$ \\
\hline Drop from ERR & 0 & $0.00 \%$ & 0 & $0.00 \%$ \\
\hline $\begin{array}{l}\text { Total Drop Via } \\
\text { Congestion }\end{array}$ & 403 & $2.00 \%$ & 249 & $1.59 \%$ \\
\hline Total Drop & 1443 & $7.15 \%$ & 702 & $4.48 \%$ \\
\hline
\end{tabular}

\section{F. Overall Summery}

This table show the overall summery of performance of proposed and previous AODV routing protocol. Each performance parameter show the improvement in case of enhanced AODV routing protocol, it means enhance the performance of network.

\begin{tabular}{|l|l|l|}
\hline PARAMETER & AODV & $\begin{array}{c}\text { AODV- } \\
\text { QoS }\end{array}$ \\
\hline Sends & 4919 & 5124 \\
\hline Receives & 4516 & 4875 \\
\hline Routing Load & 9298 & 4965 \\
\hline Packet Delivery Ratio & 91.81 & 95.14 \\
\hline Normal Routing Load & 2.06 & 1.02 \\
\hline No. of dropped data & 403 & 249 \\
\hline
\end{tabular}

Table 2 Summarize Analysis

\section{CONCLUSION}

The new design of the protocols are motivated by particular goals and requirements based on respective assumptions about the network properties or application area. Therefore, it is very important that these networks must be able to provide efficient quality of service $(\mathrm{QoS})$ that can meet the vendor requirements. To provide efficient quality of service in mobile ad-hoc networks, there is a solid need to establish new technique for routine network controls. Maintain QoS has be an important and desirable factor of MANETs. Although difficult, it is quite interesting and challenging to design and develop QoS provisioning techniques for routing in MANETs. This research provides a quality improvement in AODV routing technique to enhance their performance. The proposed routing schemes are designed based on the varying queue size at nodes of the network. Nodes with fixed queue length might cause the possibility of higher packet dropping by that we preferred to be proposed method to overcome the deficiency of long route establishment and handle the load with varying queue length. The proposed scheme definitely improves the performance of network and the improvements in this protocol are measure by performance metrics and results are proved the performance of proposed AODV QoS protocol is much better than normal AODV routing. 
In future in terms of metrics in the QoS aware routing protocols, only data rate metric is considered in the simulations. End to end delay metric could be an additional metric during the route discovery and maintenance in the routing protocol. Thus, end to end delay can be added to the AODV routing protocol.

\section{REFERENCES}

[1] C.R.Lin and J.S. Liu., "QoS routing in ad hoc wireless networks", IEEE J.Select.Areas Commun.,vol.17, pp.1488-1505, 1999.

[2] C.Perkins, "Ad-hoc On-Demand Distance Vector (AODV) routing”, RFC3561[S], 2003.

[3] D.B.Johnson, D.A.Maltz, Y.C.Hu, "The Dynamic Source Routing protocol for mobile ad hoc networks",Internet Draft, 2004.

[4] J.Hong, "Efficient on-demand routing for mobile ad hoc wireless access networks", IEEE journal on selected Areas in Communications 22(2004), 11-35.

[5] Luo Junhai, Xue Lie and Ye Danxia "Research on multicast routing protocols for mobile ad hoc networks" Computer Networks52(2008), 988-997.

[6] J. Punde, N. Pissinou, and K. Makki, “On quality of service routing in ad-hoc networks," in Proc. of LCN'03, pp. 276-278, Oct. 2003 .

[7] N. Wang and J. Chen, “A Stable On-Demand Routing Protocol for Mobile Ad Hoc Networks withWeight-Based Strategy," IEEE PDCAT’06, pp. 166-169, Dec. 2006.

[8] J. Lian., L. Li., and X. Zhu., "A Multi-Constraint QoS Routing Protocol with Route Request Selection Based on Mobile Predictibg in MANET," IEEE CISW'07, pp. 342-345, Dec. 2007.

[9] D. Kim., J. Garcia-Luna-Aceves, K. Obraczka, J.-C. Cano, and P. Manzoni, "Routing mechanisms for mobile ad hoc networks based on the energy drain rate," IEEE Transactions on Mobile Computing, vol. 2, no. 2, pp. 161-173, 2003.

[10] Omkumar. S., Rajalakshmi. S." Analysis of Quality of Service using Distributed Coordination Function in Aodv", European Journal of Scientific Research ISSN 1450-216X Vol.58 No.1, pp.6-10, 2011.

[11] P. Jacquet, et al., "Optimized Link State Routing Protocol for Ad-Hoc Networks," IEEE INMIC Multi Topic Con-ference, Lahore, December 2001, pp. 62-68.

[12] C. E. Perkins and P. Bhagwat, "Highly Dynamic Destina-tion-Sequenced Distance-Vector Routing (DSDV) for Mobile Computers," ACM press, Vol. 24, No. 4, October 1994, pp. 234-244.

[13] J. Broch, et al., "The Dynamic Source Routing Protocol for Mobile Ad-hoc Networks," IETF Internet-Draft, March 1998.

[14] C. E. Perkins, “Ad-hoc On-demand Distance Vector Rou- ting," IETF Internet-Draft, November 1997.

[15] The ns-2 home page: The Network Simulator - ns-2, http://www.isi.edu/nsnam/ns/ 